\title{
Periodic Spectrum Transmission for Single-Carrier Frequency-Domain Equalization Employing Space-Time Transmit Diversity
}

\author{
Haruhito Yoshida, Tasuku Kuriyama, Fumiaki Maehara, and Fumio Takahata \\ School of Science and Engineering, Waseda University \\ 4-1, Ohkubo 3-chome, Shinjuku-ku, Tokyo, 169-8555, Japan \\ Email: h.yoshida@toki.waseda.jp
}

\begin{abstract}
This paper presents the periodic spectrum transmission for single-carrier frequency-domain equalization (SC-FDE) employing space-time transmit diversity (STTD). The proposed scheme utilizes only even-numbered samples within a timedomain data block to create a periodical spectrum which provides frequency redundancy. Frequency redundancy is used for subchannel combining before FDE, which alleviates the loss of orthogonality caused by FDE under frequency selective fading channels. Therefore, the proposed scheme has an advantage of enhancing the overall frequency diversity benefit while keeping the space diversity benefit achieved by STTD. Computer simulation results show that the proposed scheme outperforms the typical SC-FDE using full samples over relatively severe frequency selective fading channels when applying 1-bit/sample transmission.
\end{abstract}

\section{INTRODUCTION}

In recent years, single-carrier modulation (SC) with frequency domain equalization (FDE) has received a lot of interest because SC modulation essentially makes it possible to reduce the peak-to-average transmitted power ratio (PAPR) which is the major problem in OFDM. Furthermore, this approach has an advantage of exploiting the frequency diversity benefit without bandwidth expansion and without decreased data rate because each bit is simultaneously modulated over all sub-channels [1]-[4].

On the other hand, the antenna diversity is well-known as an effective approach to create the space diversity effect. In particular, space-time transmit diversity (STTD) has an attractive feature which provides the diversity effect for two transmit antennas without both the transmission rate reduction and the channel state information (CSI) at the transmitter [5]. As for SC-FDE employing STTD, an STTD encoding only in the time domain signal processing has been introduced and its performance is evaluated in [6].

So far, we have proposed the application of the periodic spectrum to SC-FDE for single antenna system with the aim of enhancing the frequency diversity benefit [7]. The feature of this approach is to generate the periodic spectrum by using the even-numbered time domain samples within a block, which makes it possible to create frequency redundancy used for frequency diversity before FDE and consequently to enhance the overall frequency diversity effect. Thus, as an extension of this previous work, it would be interesting to investigate the effect of the periodic spectrum on STTD.

In this paper, we propose the periodic spectrum transmission for SC-FDE employing space-time transmit diversity. In the proposed scheme, the total diversity benefit can be obtained by FDE of the transmitting periodical spectrum, as well as STTD. Here, it should be noted that, since the proposed scheme causes the data rate reduction due to the decreased samples within a block, the high-level modulation has to be applied so as to keep the constant rate transmission. The effectiveness of the proposed scheme is demonstrated compared with the typical SC-FDE using full samples with parameters of the delay spread, the fading correlation between two antenna branches, and the transmission rate.

The following section describes the system configuration of the proposed scheme. Section III shows the performance comparison between the proposed scheme and the typical SCFDE. Finally, Section IV summarizes this paper.

\section{Proposed ScHEME}

\section{A. Periodic spectrum transmission for SC-FDE}

The frequency spectrum of the SC modulated signal is expressed as

$$
X(k)=\sum_{n=0}^{N-1} x(n) e^{-j 2 \pi\left(\frac{n}{N}\right) k}
$$

where $N$ is a size of discrete Fourier transform (DFT) and $x(n)$ is the SC modulated sample in the time domain. Thus the frequency spectrum of the SC modulated signal using only even-numbered samples is given by

$$
X(k)=\sum_{i=0}^{N / 2-1} x(2 i) e^{-j 2 \pi\left(\frac{2 i}{N}\right) k}
$$

When $N^{\prime}$ is defined as $N / 2$, Eq. (2) can be rewritten in the following form:

$$
X(k)=\sum_{i=0}^{N^{\prime}-1} x(2 i) e^{-j 2 \pi\left(\frac{i}{N^{\prime}}\right) k} .
$$

From Eq. (3), it can be seen that it has a periodical frequency spectrum with the period of $N^{\prime}$. 
Full samples

( $\mathrm{N}$ samples within a block)
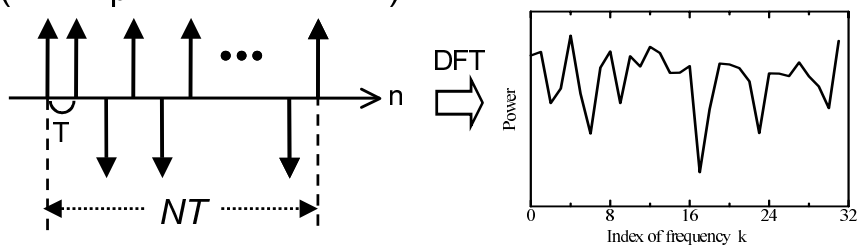

Only even-numbered samples (N/2 samples within a block)
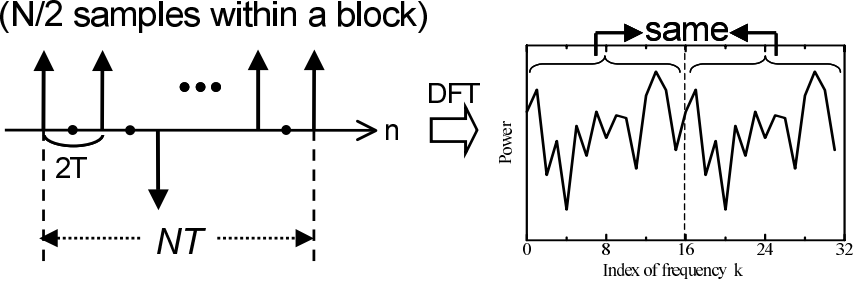

Fig. 1. Comparison of frequency spectrums between full samples and only even-numbered samples $(N=32)$.

Figure 1 illustrates an example of the frequency spectrum for $N=32$. From Fig. 1, it is apparent that the frequency spectrum with only even-numbered samples is periodic while the spectrum with the full samples has no periodicity. This fact implies that the SC modulated signal using only evennumbered samples is capable of creating the frequency redundancy, and its redundancy can be used for frequency diversity.

\section{B. STTD Encoding for SC-FDE}

In this paper, Alamouti's STTD encoding [5], where the number of transmit branches is two, is applied to both the proposed scheme using only even-numbered samples and the typical SC-FDE using full samples in the frequency domain. When $X_{p}(k)$ is the sub-channel signal of the $p$-th DFT block, STTD encoding is performed over two consecutive data blocks $(p=0,1)$ in a sub-channel by sub-channel manner as shown in Table I, where $(.)^{*}$ denotes complex conjugate operation. Since STTD including the complex conjugate operation is conducted in the frequency domain, the signal processing in the frequency domain are originally required by both the $N$-point DFT and Inverse DFT (IDFT). However, the complex conjugate operation in the frequency domain fortunately corresponds to performing time domain samples in reverse order and is given by

$$
\frac{1}{N} \sum_{k=0}^{N-1} X_{p}^{*}(k) e^{j 2 \pi\left(\frac{k}{N}\right) n}=x_{p}^{*}(N-n) .
$$

Therefore, the complex conjugate operation of sub-channel signals in a block is implemented only by time domain signal processing. Figure 2 shows STTD encoding process in the time domain. As shown in this figure, in practice, STTD encoding can be realized without the FFT and IFFT processing at the transmitter [6].

\section{System Configuration}

Figure 3 shows a configuration of the proposed SC-FDE scheme. At the transmitter, incoming information bits are
TABLE I

STTD ENCODED SIGNAL $S_{l, p}(k)$ OF THE $k$-TH SUB-CHANNEL.

\begin{tabular}{c|cc}
\hline \multirow{2}{*}{ Block index $(p)$} & \multicolumn{2}{|c}{ Antenna index $(l)$} \\
\cline { 2 - 3 } & 0 & 1 \\
\hline 0 & $X_{0}(k)$ & $X_{1}(k)$ \\
\hline 1 & $-X_{1}^{*}(k)$ & $X_{0}^{*}(k)$ \\
\hline
\end{tabular}

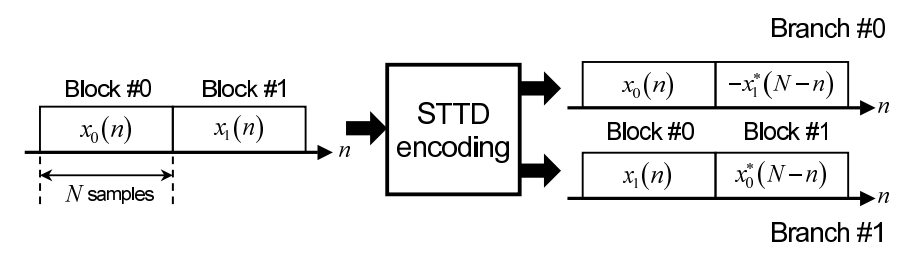

Fig. 2. STTD encoding process in the time domain.

mapped into the transmit data symbols $x(n)$ at the sampling period of $2 T$. Here, it is noted that, since $2^{2 m}$-ary modulation is adopted in the case of $m$-bit/sample transmission for constant rate transmission, each sample power can be enhanced to twice as much as that of the typical SC with the sampling period of $T$. For example, considering 1-bit/sample transmission, QPSK and BPSK are chosen as the proposed scheme and the typical SC scheme, respectively. Next, STTD encoding converts the consecutive data blocks into the blocks for two transmit branches, which can be realized without the FFT and IFFT processing at the transmitter as shown in Section II-B. After the guard interval insertion, these signals are transmitted simultaneously from two antennas.

At the receiver, the effective SC symbol is extracted by removing the guard interval with the length of $T_{G}$. The received samples in the time domain are fed into the $N$-point FFT circuit through S/P converters and are converted into the $N$ sub-channel signals. The $k$-th sub-channel signal of the $p$-th block $R_{p}(k)$ is given by

$$
R_{p}(k)=\sum_{l=0}^{1} H_{l}(k) S_{l, p}(k)+N_{p}(k)
$$

where $S_{l, p}$ and $H_{l}(k)$ are the transmitted signal and the channel frequency response from transmit antenna $l$ respectively, and $N_{p}(k)$ denotes AWGN. Here, it is assumed that the channel variation within two blocks is constant. Two consecutive received sub-channel blocks are simultaneously recovered by STTD decoding, which achieves the maximal ratio combining (MRC) gain in a sub-channel by sub-channel manner:

$$
\begin{aligned}
& Y_{0}(k)=H_{0}^{*}(k) R_{0}(k)+H_{1}(k) R_{1}^{*}(k) \\
& Y_{1}(k)=H_{1}^{*}(k) R_{0}(k)-H_{0}(k) R_{1}^{*}(k) .
\end{aligned}
$$

Taking advantage of the periodic characteristic of the transmitting spectrum, the proposed scheme can combine two subchannel signals with the frequency interval of $N^{\prime}$ according to a maximal ratio, which results in $N^{\prime}$ sub-channel signals. Hence, the signal of the $k$-th sub-channel in the $p$-th block 


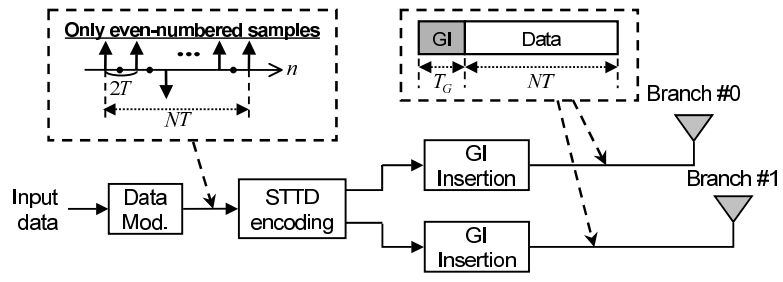

(a) Transmitter

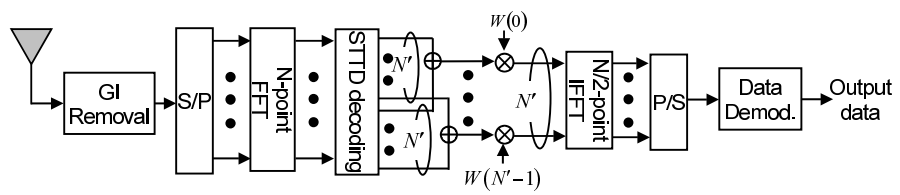

(b) Receiver

Fig. 3. Configuration of proposed SC-FDE scheme.

after MRC combining is expressed as

$$
\begin{gathered}
\hat{Y}_{p}(k)=Y_{p}(k)+Y_{p}\left(k+N^{\prime}\right) \\
\quad \text { for } k=0,1, \ldots, N^{\prime}-1 .
\end{gathered}
$$

After MRC combining, each sub-channel signal is multiplied by an appropriate weighting factor $W(k)$ to reduce the inter-bit interference because the frequency selective fading introduces the loss of orthogonality among the different data samples which spread over $N^{\prime}$ sub-channels [3]. The weighted signal of the $k$-th sub-channel is given by

$$
\hat{X}_{p}(k)=W(k) \hat{Y}_{p}(k) .
$$

In the proposed scheme, $W(k)$ is chosen to satisfy the minimum mean square error (MMSE) criterion because MMSE can minimize the interference among samples and noise. The MMSE-based weight of the $k$-th sub-channel after both STTD decoding and MRC is expressed by

$$
\begin{gathered}
W(k)=\frac{1}{N^{\prime} \sum_{l=0}^{1}\left(\left|H_{l}(k)\right|^{2}+\left|H_{l}\left(k+N^{\prime}\right)\right|^{2}\right)+\sigma_{n}^{2}} \\
\quad \text { for } k=0,1, \ldots, N^{\prime}-1
\end{gathered}
$$

where $\sigma_{n}^{2}$ is the variance of AWGN.

Then the weighted sub-channel signals are fed to $N / 2$-point IFFT circuit and are transformed into the time domain signals. In other words, the aim of IFFT processing here is actually to combine coherently the sub-channel signals separated in advance at the transmitter. The $n$-th data sample in the $p$-th block is given by

$$
\begin{gathered}
\hat{x}_{p}(n)=\frac{1}{N^{\prime}} \sum_{k=0}^{N^{\prime}-1} \hat{X}_{p}(k) e^{j 2 \pi\left(\frac{k}{N^{\prime}}\right) n} \\
\text { for } n=0,1, \ldots, N^{\prime}-1 .
\end{gathered}
$$

Finally, the time domain data samples are quantized to recover the transmitted data sequence.
TABLE II

SIMULATION PARAMETERS.

\begin{tabular}{c|c}
\hline $\begin{array}{c}\text { Modulation } \\
(m=1)\end{array}$ & $\begin{array}{c}\text { BPSK (Full samples) } \\
\text { QPSK (Proposed) }\end{array}$ \\
\hline Detection & Coherent detection \\
\hline FFT size $(N)$ & 32 \\
\hline Number of sub-channels $\left(N_{c}\right)$ & 32 \\
\hline Guard interval length $\left(T_{G}\right)$ & 8 samples \\
\hline Channel model & 6-ray exponentially \\
& -decaying model \\
\hline Channel estimation & perfect \\
\hline
\end{tabular}

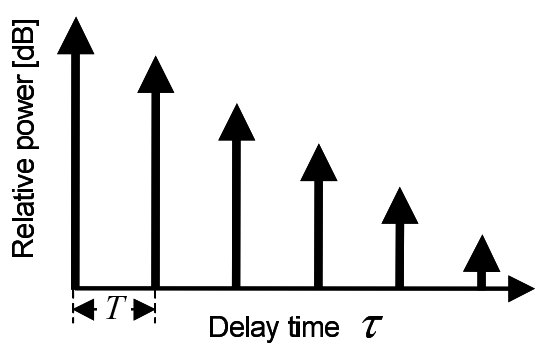

Fig. 4. Radio channel model.

\section{PERformance Evaluation}

\section{A. Simulation parameters}

The simulation parameters are given in Table II. As shown in this table, firstly, $m=1$ is considered for the numerical evaluation. Then, $m \geq 2$ is considered in Section III-E. Figure 4 shows the radio channel model, where 6-path exponentiallydecaying multipath channels are assumed. The path separation is set to be the sampling period $T$, and the amplitude and phase of each ray are characterized by Rayleigh distribution and uniform distribution, respectively. Here, it is noted that the duration of the guard interval $T_{G}$ is longer than the channel maximum delay spread and therefore no intersymbol interference (ISI) occurs. In our simulations, no carrier frequency offset and ideal clock synchronization are assumed, and the channel variation within two consecutive blocks is not taken into account. Moreover, the channel estimation is assumed to be perfect.

\section{B. BER versus Average CNR $\Gamma$}

Figure 5 shows the performance comparison, in terms of the Bit Error Rate (BER) versus the average CNR $\Gamma$, between the proposed scheme ("Proposed") and the typical STTD-SCFDE using full samples ("Full samples") with a parameter of the delay spread $\tau_{r m s}$, where $m=1$. The theoretical BER performance of STTD under frequency non-selective Rayleigh fading channels is also shown for reference.

It can be found from Fig. 5 that the flat fading channels such as $\tau_{r m s}=0$ lead to the same BER between the proposed scheme and the typical STTD-SC-FDE. In addition, the BER performances of both schemes correspond to the theoretical BER. This result comes from two reasons. One is that QPSK with the power enhancement of 3-dB provides 


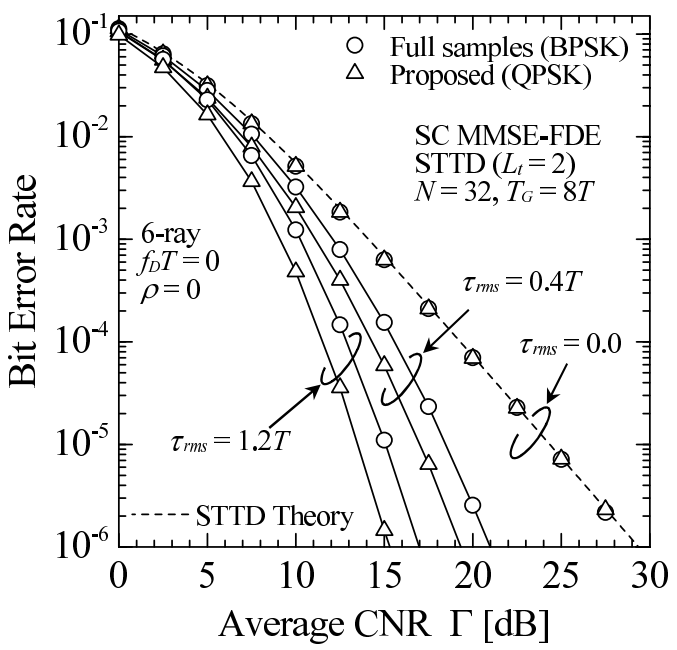

Fig. 5. Performance comparison, in terms of BER versus average CNR $\Gamma$, between the proposed scheme and the typical STTD-SC-FDE, where $m=1$.

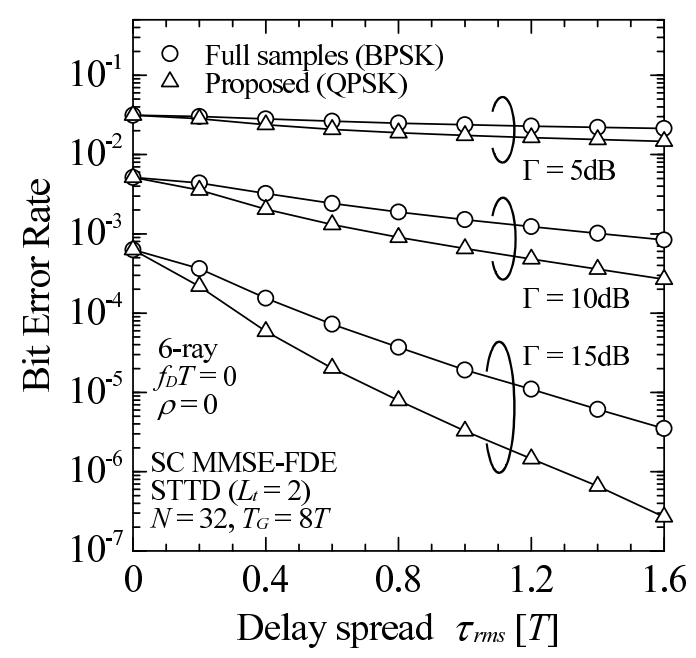

Fig. 6. Performance comparison, in terms of BER versus delay spread $\tau_{r m s}$, between the proposed scheme and the typical STTD-SC-FDE, where $m=1$. the same BER as BPSK in general and the other is that the flat fading channels do not create the frequency diversity benefit irrespective of the scheme.

On the other hand, it can be observed that, at $\tau_{r m s}=0.4 T$ and $1.2 T$, the proposed scheme has better performance than the typical STTD-SC-FDE. This is because, in the proposed scheme, the signal combining of two sub-channels before FDE relaxes the loss of orthogonality due to the frequency selective fading, which consequently enhances the overall frequency diversity effect.

\section{BER versus Delay Spread $\tau_{r m s}$}

Figure 6 shows the performance comparison, in terms of the BER versus the delay spread $\tau_{r m s}$, between the proposed scheme and the typical STTD-SC-FDE using full samples with a parameter of the average $\mathrm{CNR} \Gamma$, where $m=1$. It is found from Fig. 6 that, irrespective of $\Gamma$, the BER performances of both schemes are improved with the increase in $\tau_{r m s}$. The reason is that the increase in $\tau_{r m s}$ gives more frequency diversity benefit regardless of the scheme.

Moreover, as $\tau_{r m s}$ increases, the proposed scheme can provide better BER performance than the typical STTD-SCFDE regardless of $\Gamma$. This is because, in the proposed scheme, the signal combining of two sub-channels before FDE relaxes the loss of orthogonality due to the frequency selective fading, which results in the enhancement of the total diversity effect as discussed in Section III-B.

\section{BER versus Fading Correlation between Two Branches $\rho$}

Figure 7 shows the performance comparison, in terms of the BER versus space correlation $\rho$, between the proposed scheme and the typical STTD-SC-FDE using full samples with a parameter of the delay spread $\tau_{r m s}$, where the average $\mathrm{CNR}$ $\Gamma=15 \mathrm{~dB}$ and $m=1$. The theoretical BER performance of
STTD under frequency non-selective Rayleigh fading channels is also shown for reference.

From Fig. 7, it can be seen that, regardless of $\tau_{r m s}$, the BER performances of both schemes are degraded with the increase of $\rho$. The reason is that the space diversity benefit created by STTD decreases as $\rho$ increases. Here, it is interesting to note that both schemes give the same performance in the case of $\tau_{r m s}=0$. This is because the BER performance is only dependent on the space diversity benefit in this case.

On the other hand, in the case of $\tau_{r m s}=0.4 T$ and $1.2 T$, the proposed scheme provides better BER performance than the typical SC-FDE regardless of $\rho$. Under such conditions, since the space diversity is the same for both schemes, the difference in the BER between two schemes is caused by how much the frequency diversity effect can be exploited in each scheme.

\section{E. Required Average CNR versus Transmission Rate $m$}

In this section, the influence of the number of bits per one sample $m$ on the required average CNR at a certain BER is evaluated and discussed. Table III shows the possible modulations for both the proposed scheme and the typical SC scheme. As shown in this table, the modulation level has to be higher, especially in the proposed scheme, with the increase in $m$. In detail, the proposed scheme adopts $2^{2 m}$-ary modulation when $m$-bit/sample transmission is applied to the typical SC scheme.

Figure 8 shows the performance comparison, in terms of the transmission rate $m$ versus the required average CNR in order to satisfy the BER $=10^{-5}$, between the proposed scheme and the typical STTD-SC-FDE, where the delay spread $\tau_{r m s}=1.2 T$. From this Fig. 8, it is found that, for 1-bit transmission, the proposed scheme can decrease the required average CNR by about $1.4 \mathrm{~dB}$ and $4.0 \mathrm{~dB}$ in the case of $\rho=0.0$ and 1.0, respectively. However, as $m$ increases, the required 


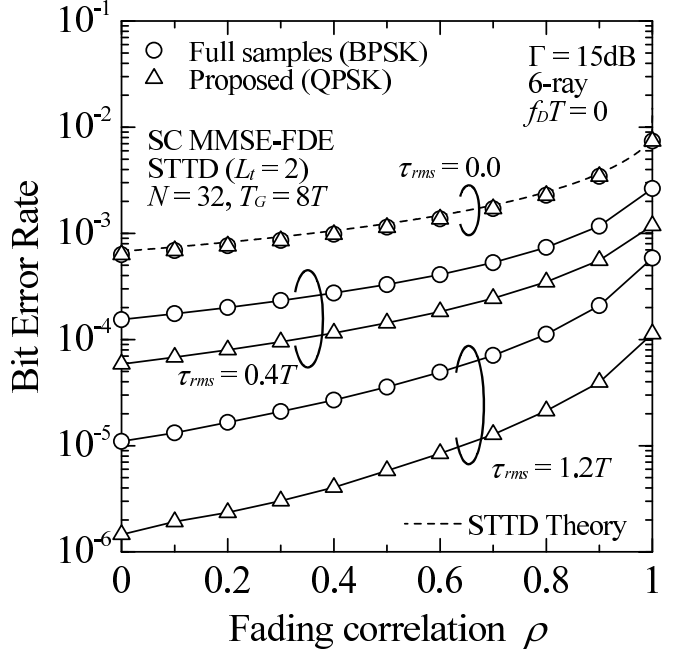

Fig. 7. Performance comparison, in terms of BER versus fading correlation between two branches $\rho$ between the proposed scheme and the typical STTDSC-FDE, where $m=1$.

TABLE III

POSSIBLE MODULATIONS FOR TWO DIFFERENT SCHEMES.

\begin{tabular}{c|cccc}
\hline bits/sample $(m)$ & 1 & 2 & 3 & 4 \\
\hline Full samples & BPSK & QPSK & 8PSK & 16QAM \\
\hline Proposed & QPSK & 16QAM & 64QAM & 256QAM \\
\hline
\end{tabular}

average CNR of the proposed scheme is the same or larger in some degree compared with the typical STTD-SC-FDE. This is because the inter-bit interference due to the use of the high-level modulation cannot be neglected with the increase in $m$, which degrades the effect of FDE. Therefore, it can be concluded that the proposed approach is effective in the low rate transmission such as 1-bit/sample transmission, while the typical STTD-SC-FDE is superior to the proposed scheme in the relatively high rate transmission.

\section{CONCLUSION}

We have proposed the periodic spectrum transmission for SC-FDE employing STTD. The proposed scheme provides the space and frequency diversity benefits and only evennumbered samples within a time-domain SC data block create the frequency redundancy used for enhancing the overall frequency diversity benefit. Computer simulation results showed that, when applying 1-bit/sample transmission, the proposed scheme outperforms the typical STTD-SC-FDE using full samples over relatively severe frequency selective fading channels, while the typical STTD-SC-FDE provides better BER than the proposed scheme in the relatively high rate transmission.

\section{REFERENCES}

[1] V. Aue, R. Valenzuela, and G. P. Fettweis, "A comparison of the performance of linearly equalized single carrier and coded OFDM over frequency-selective fading channels using the random coding technique," Proc. of IEEE ICC '98, vol.2, pp.753-757, June 1998.

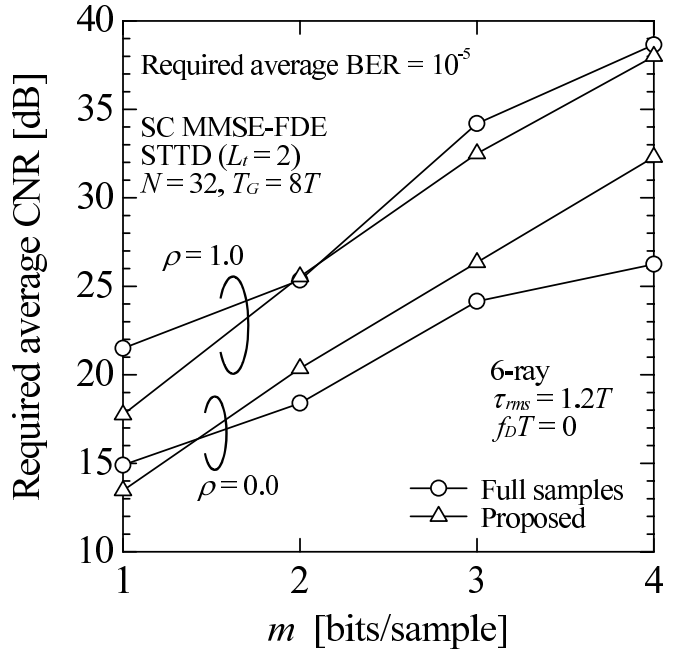

Fig. 8. Performance comparison, in terms of required average CNR versus transmission rate $m$ between the proposed scheme and the typical STTD-SCFDE.

[2] M. V. Clark, "Adaptive frequency-domain equalization and diversity combining for broadband wireless communications," IEEE Journal on Selected Areas in Communications, vol.16, pp.1385-1395, Oct. 1998.

[3] D. Falconer, S. L. Ariyavisitakul, A. Benyamin-Seeyar and B. Eidson, "Frequency domain equalization for single-carrier broadband wireless systems," IEEE Communications Magazine, vol.40, pp.58-66, April 2002.

[4] F. Maehara and H.-P. Kuchenbecker, "Performance comparison of carrier interferometry OFDM and OFDM using space diversity reception and channel coding," IEICE Trans. Commun., Vol.E87-B, No.12, pp.38013808, Dec. 2004.

[5] S.M. Alamouti, "A simple transmit diversity technique for wireless communications," IEEE Journal on Selected Areas in Communications, vol.16, no.8, pp.1451-1458, Oct. 1998.

[6] N. Al-Dhahir, "Single-carrier frequency-domain equalization for spacetime block-coded transmissions over frequency-selective fading channels," IEEE Commun., Letters, Vol.5, Issue 7, pp304-306, July 2001.

[7] F. Maehara and H.-P. Kuchenbecker, "Frequency diversity application to single-carrier transmission with frequency-domain equalization," Proc. WPMC 2004, Vol.1, pp.324-328, Sept. 2004. 